MATEC Web of Conferences 22,01026 (2015)

DOI: $10.1051 /$ matec conf/20152201026

(C) Owned by the authors, published by EDP Sciences, 2015

\title{
A Method for Image Decontamination Based on Partial Differential Equation
}

\author{
Junping Hou \\ Mathematics and Physics Department, North China Electric Power University, Baoding, Hebei, China
}

\begin{abstract}
This paper will introduce the method to apply partial differential equations for the decontamination processing of images. It will establish continuous partial differential mathematical models for image information and use specific solving methods to conduct decontamination processing to images during the process of solving partial differential equations, such as image noise reduction, image denoising and image segmentation. This paper will study the uniqueness of solution for the partial differential equations and the monotonicity that functional constrain has on multipliers by making analysis of the ROF model in the partial differential mathematical model
\end{abstract}

Keywords: image denoising; partial differential equation; PDE; wavelet model; Fourier transform

\section{INTRODUCTION}

People always process images and make analysis of other processing methods. Nowadays, with the rapid development in the application of electronic equipment, such as computers, the transfer of images has become more convenient. However, during the process of transfer, the original images frequently become fuzzy or get reversed which may cause mistakes in image information. Especially in the areas of medical science, military affairs and engineering, image damage can bring serious results

In fact, image decontamination is the processing of image signals, such as image denoising and image damage removal. Traditional image denoising is to apply wavelet analysis and Fourier analysis. However, there're certain narrow limitations existing in these two methods. They both have requirements for the stationarity and time-frequency duality of the signals.

Partial differential equation is a new means for image denoising. It considers the geometrical features of image signals on the basis of building mathematical models. Nevertheless, the solving of partial differential equation has certain restrictions due to the infinite solutions of partial differential equation numerical solutions which may occur because of massive image information.

\section{PARTIAL DIFFERENTIAL EQUATION}

Partial differential equation is a science of mathematics applied in engineering modeling. However, with the extension of its application range, partial differential equation has made new progress in physical chemistry area. Nowadays, partial differential equation has obtained development in many other new areas, such as finance and image processing. In basic subjects, partial differential equation refers to the solving process of mathematical theories, such as calculus of variations, ordinary differential equation and theory of functions. Within its extension in other subjects, partial differential equation can become an independent research field.

If the normal form of the differential equation that involves an n-member function $\varphi=\varphi\left(x_{1}, x_{2}, x_{3}, \cdots, x_{n}\right)$ is as follows:

$$
F\left(x_{1}, x_{2}, x_{3}, \cdots, x_{n}, \varphi, D \varphi_{1}, D^{2} \varphi_{2}, \cdots, D^{m} \varphi_{n}\right)=0
$$

Among which, $D^{k} \varphi=\frac{\partial^{k} \varphi}{\partial x_{1}^{k_{1}} \partial x_{2}^{k_{2}} \cdots \partial x_{n}^{k_{n}}}$ and

$\sum_{i=1}^{n} k_{i}=k, k=1,2, \cdots, 3$

The second order differential equation should be:

$\sum_{i, j=1}^{n} a_{i j} \frac{\partial^{2} \varphi}{\partial x_{i} \partial x_{j}}+\sum_{i=1}^{n} b_{j} \frac{\partial \varphi}{\partial x_{i}}+c \varphi=g$

Among which, $a_{i j}, b_{j}, c$ and $g$ are the functions of $x_{1}, x_{2}, x_{3}, \cdots, x_{n}$.

Definite Condition

Generally speaking, partial differential equation is a form of expression of the equation expressing the phenomena of the same kind that occur in some physical phenomenon. For a partial differential equation, its solutions can be infinite. However, among the infinite solutions, seeking out the universal law of the solutions cannot solve the essence of the problems. An issue under a special condition must be selected and the condition is the definite condition of the partial differential equation. 
MATEC Web of Conferences

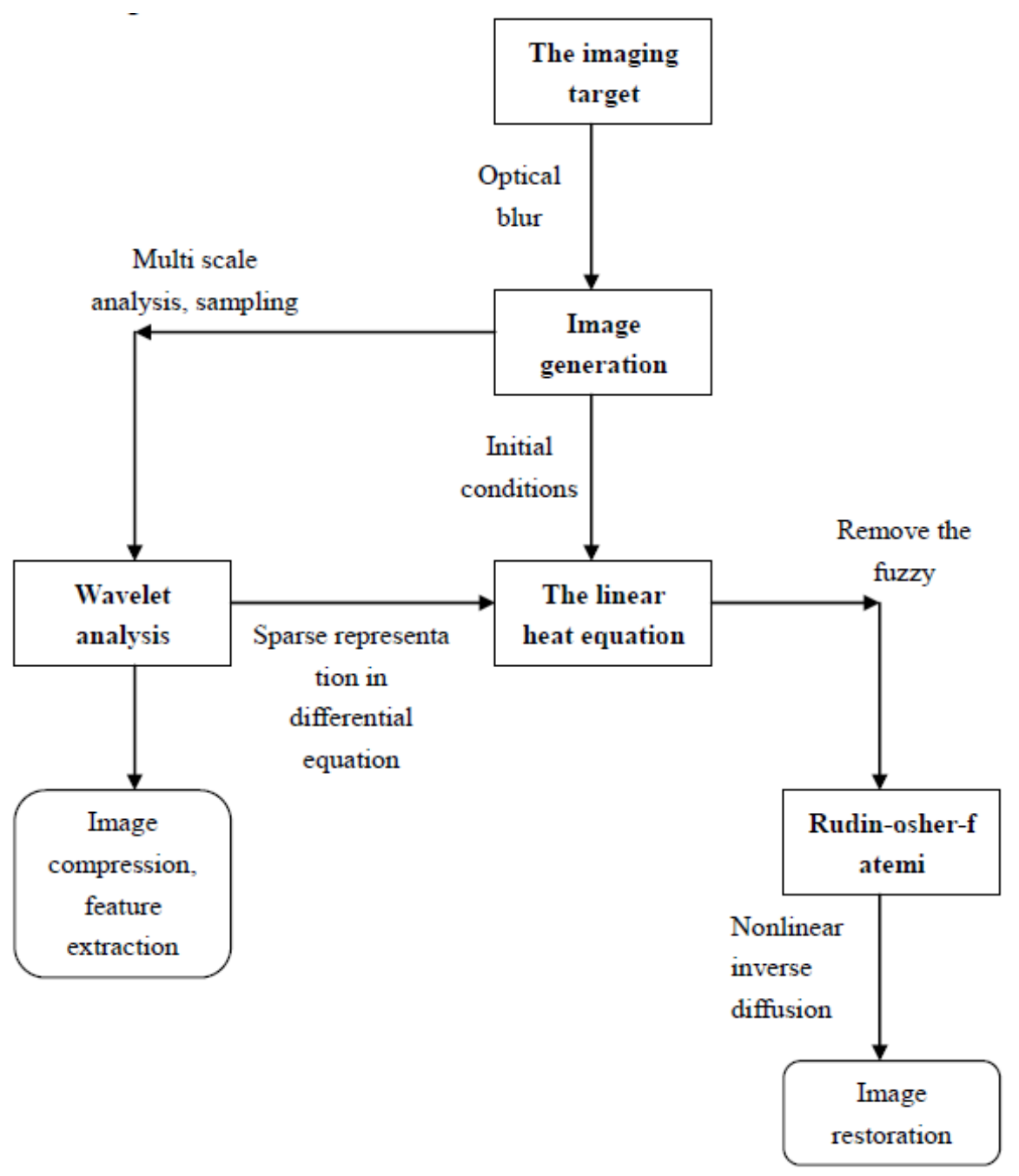

Figure 1. The Process of Solving Image Damage by Partial Differential Equation (1)

Condition of Boundary Value:

The first condition of boundary value: describe the direct physical quantity of the boundary system.

$\left.\varphi(x, t)\right|_{x \in \partial \Omega}=\psi_{1}(x, t), t \geq 0$

The second condition of boundary value: the reciprocal value of the direct physical quantity in the familiar boundary system, such as heat transfer and conduction:

$\left.\frac{\partial \varphi(x, t)}{\partial n}\right|_{x \in \partial \Omega}=\psi_{1}(x, t), t \geq 0$

Where, $\stackrel{1}{n}$ refers to the unit normal vector of $\partial \Omega$.

The third condition of boundary value: describe the combing form of the direct physical quantity in the boundary system and the reciprocal of this physical quantity: $\left.\left(\frac{\partial \varphi(x, t)}{\partial n}+\alpha \varphi\right)\right|_{x \in \partial \Omega}=\psi_{1}(x, t), t \geq 0$

Initial Value Condition:

In the equations above, besides the partial derivative of spatial variable, the partial derivative of time should also be obtained. Therefore, the setting of initial values is required in some partial differential equations, so as to make it more convenient for equation solving.

\section{SOLUTIONS FOR IMAGE DECONTAMINA- TION BASED ON PARTIAL DIFFERENTIAL EQUATION}

The approach to apply partial differential equation in restoring images is actually to do image morphing fist 


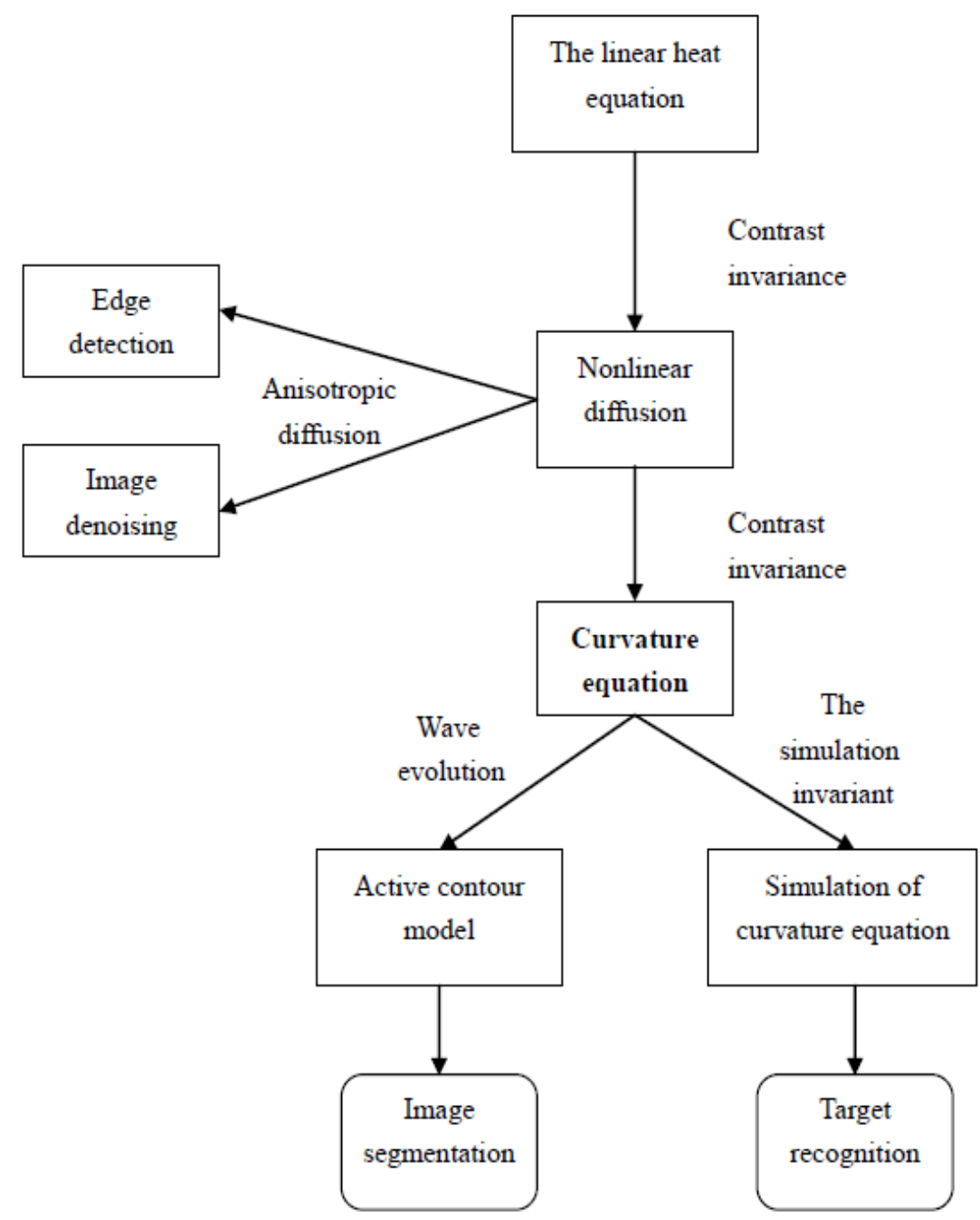

Figure 2. The Process of Solving Image Damage by Partial Differential Equation(2)

and then solve the partial differential equation. The related modeling process is shown in Figure 1.

During the processes of image formation and image transfer, the generation of noise is inevasible. In some special conditions, high level of noise can cause serious problems. Hence, image denoising is an important part in image processing procedures.

Denoising refers to the removal of noise and vibration from a damaged image with the condition of reserving the original image features. At present, there are three main approaches to complete image denoising:

(1) Wavelet and Fourier variation approach;

(2) PDE and variation approach;

(3) Statistical approach similar to MRF.

Among the approaches above, the PDE partial differential equation has better application in image denoising. The reason for this phenomenon is that PDE can effectively reduce image noise and image vibration under the condition of maintaining the basic features of the original image.

\section{ESTABLISHMENT OF ROF MODEL}

Suppose $z\left(x, x_{2}\right): \Omega \rightarrow R^{+}$is the image with noise; $\Omega$ is the bounded raised image region existing in $R^{2} ; u\left(x_{1}, x_{2}\right)$ is the original image; and $\eta\left(x_{1}, x_{2}\right)$ is the noise that appeared.

$z\left(x_{1}, x_{2}\right)=u\left(x_{1}, x_{2}\right)+\eta\left(x_{1}, x_{2}\right)$

The ROF model is small in form:

$\min _{u} T V(u)=\int_{\Omega}|\nabla u| d x$ s.t 


\section{MATEC Web of Conferences}

$F(u)=\frac{1}{2}\left(\|u-z\|_{L^{2}(\Omega)}^{2}-\sigma^{2}\right)=0$

The $L^{2}(\Omega)$-norm of noise data:

$\sigma=\left(\int_{\Omega}\left|z\left(x_{1}, x_{2}\right)-\mu\left(x_{1}, x_{2}\right)\right|^{2} d x\right)^{\frac{1}{2}}$

$\sigma$ is a known priori equation. The objective function $T V(u)$ is non-differentiable when $|\nabla u|=0$. Therefore, the following equations should be considered:

$$
\begin{aligned}
& \min _{u} T V(u)=\int_{\Omega} \sqrt{|\nabla u|^{2}+\varepsilon} d x \\
& F(u)=\frac{1}{2}\left(\|u-z\|_{L^{2}(\Omega)}^{2}-\sigma^{2}\right)=0
\end{aligned}
$$

Among which, $\varepsilon>0$ is a very small smooth coefficient.

The expression of the lagrange function that restrains total variation optimization problem is given below:

$$
E(\lambda, u)=\int_{\Omega} \sqrt{|\nabla u|^{2}+\varepsilon} d x+\frac{\lambda}{2}\left(|u-z|^{2} d x-\sigma^{2}\right)
$$

Among which, $\lambda$ is the multiplier of lagrange. In consequence, the optimization model of ROF is as follows:

$$
\min _{\substack{u \in B /(\Omega) \\ \lambda \in R}} E(\lambda, u)
$$

Proceed to first-order variation and export the optimum condition as follows:

$$
\begin{aligned}
& L_{\lambda}(u)=-\nabla \cdot\left(\frac{\nabla u}{\sqrt{|\nabla u|^{2}+\varepsilon}}\right)+\lambda(u-z)=0 \\
& F(u)=\frac{1}{2}\left(\|u-z\|_{L^{2}(\Omega)}^{2}-\sigma^{2}\right)=0
\end{aligned}
$$

It should satisfy the boundary condition that $u \in \kappa=\left\{u\left(x_{1}, x_{2}\right):\left(x_{1}, x_{2}\right) \in \Omega\right.$ and $\frac{\partial u(x, t)}{\partial n}=0$ on $\left.\partial \Omega\right\}$

Where, $\stackrel{1}{n}$ refers to the unit normal vector of $\partial \Omega$.

\section{SOLUTION FOR ROF MODEL BASED ON PARABOLIC-TYPE EVOLUTION ALGO- RITHM}

Use classical parabolic-type evolution method to solve the equation (3.6a):

$\frac{\partial u(\cdot, t)}{\partial t}=N(u)=\nabla \cdot\left(\frac{\nabla u(\cdot, t)}{\sqrt{|\nabla u(\cdot, t)|^{2}+\varepsilon}}\right)-\lambda(u(\cdot, t)-u(\cdot, 0))$
Among which, $t>0$.

$u\left(x_{1}, x_{2}, 0\right)=z\left(x_{1}, x_{2}\right) \quad, \quad\left(x_{1}, x_{2}\right) \in \Omega \quad$,

$u(\cdot, t) \in \kappa \times R^{+}$

Iteration of lagged diffusion fixed points

Use Newton's linearizing techniques to take parabolic-type evolution algorithm into consideration:

$$
\begin{aligned}
L_{\lambda}^{\prime}\left(u^{n}\right)\left(u^{n+1}-u^{n}\right)= & -\nabla \cdot\left(\frac{\nabla u\left(u^{n+1}-u^{n}\right)}{\sqrt{\left.\nabla u^{n}\right|^{2}+\varepsilon}}\right) \\
& +\nabla \cdot\left(\frac{\nabla u\left(u^{n+1}-u^{n}\right)^{T} \nabla u^{n}}{\left(\sqrt{\left.\nabla u^{n}\right|^{2}+\varepsilon}\right)^{3}} \nabla u^{n}\right)+\lambda\left(u^{n+1}-u^{n}\right) \\
& =-L_{\lambda}\left(u^{n}\right)
\end{aligned}
$$

which can be simplified as:

$\lambda u^{n+1}-\nabla \cdot\left(\frac{\nabla u\left(u^{n+1}-u^{n}\right)}{\sqrt{\left|\nabla u^{n}\right|^{2}+\varepsilon}}\right)+\nabla \cdot\left(\frac{\nabla u\left(u^{n+1}-u^{n}\right)^{T} \nabla u^{n}}{\left(\sqrt{\left|\nabla u^{n}\right|^{2}+\varepsilon}\right)^{3}} \nabla u^{n}\right)$

$+\lambda\left(u^{n+1}-u^{n}\right)=\lambda z$

As the equation above has divergence when $\varepsilon$ is very small, a solution with certain truth is required to ensure the convergence of the equation. Therefore, the following equation appears:

$\lambda u^{n+1}-\nabla \cdot\left(\frac{\nabla u^{n+1}}{\sqrt{\left|\nabla u^{n}\right|^{2}+\varepsilon}}\right)=\lambda z$

Alternative Split-Bregman Iterative Method

Alternative split-bregman iterative method is used to solve the reply issues of discrete images.

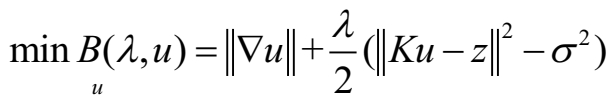

Among which, $K$ refers to the fuzzy operator,

$\left\{\begin{array}{l}u=\arg \min _{u} \frac{u}{2}\|K u-z+c\|_{2}^{2}+\frac{\lambda}{2}\|D u-d+b\|_{2}^{2} \\ d=\arg \min _{u}\|d\|^{2}+\frac{\lambda}{2}\|d-D u+b\|^{2} \\ b=b+\delta_{b}(D u-d) \\ c=c+\delta_{c}(K u-z)\end{array}\right.$

Where, $u>0 ; 0<\delta_{b} \leq 1 ; 1<\delta_{c}<2 ;$ and $D u=T V(u)$.

In a two-dimensional image, $u$ can be set as 


\section{ICETA 2015}

$u\left(x_{1}, x_{2}, t\right)$ and time can be set as $t_{n}=n \Delta t$. Within the range of $\left(i, j, t_{n}\right)$, use the center finite difference method to obtain the reciprocal that $u$ generates with spatial variables; and apply the forward difference method to obtain the reciprocal of time. The obtained results are as follows:

$$
\begin{aligned}
& \left(u_{x}\right)_{i, j}^{n}=\frac{u_{i+1, j}^{n}-u_{i-1, j}^{n}}{2 h} \\
& \left(u_{y}\right)_{i, j}^{n}=\frac{u_{i+1, j}^{n}-u_{i, j-1}^{n}}{2 h} \\
& \left(u_{x x}\right)_{i, j}^{n}=\frac{u_{i+1, j}^{n}-2 u_{i j}^{n}+u_{i-1, j}^{n}}{h^{2}} \\
& \left(u_{y y}\right)_{i, j}^{n}=\frac{u_{i+1, j}^{n}-2 u_{i j}^{n}+u_{i, j-1}^{n}}{h^{2}} \\
& \left(u_{x y}\right)_{i, j}^{n}=\frac{u_{i+1, j+1}^{n}-u_{i-1, j+1}^{n}+u_{i+1, j-1}^{n}+u_{i-1, j-1}^{n}}{4 h^{2}} \\
& \left(u_{t}\right)_{i, j}^{n}=\frac{u_{i, j}^{n}-u_{i, j}^{n}}{\Delta t}
\end{aligned}
$$

Where, $h$ refers to the step size of a two-dimensional grid and $i, j$ respectively refer to the corresponding values of $x, y$.

\section{TEST IMAGE}

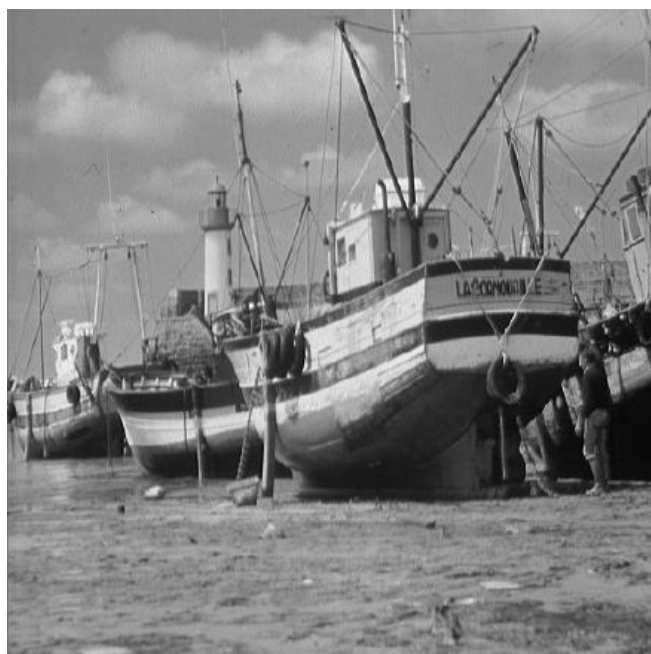

Figure 3. Original Picture
The original picture is shown in Figure 3. Figure 4 shows the comparison picture after noise adding (with $50 \%$ data lost) while Figure 5 shows the image after denoising. Establish the following parameters: time step $\Delta t=0.1$; number of iterations is 25 times; normalization parameter $\varepsilon=0.05$; and constrained parameter $\beta=0.4$. It can be seen that the image has much better effect after being processed by partial differential equation denoising.

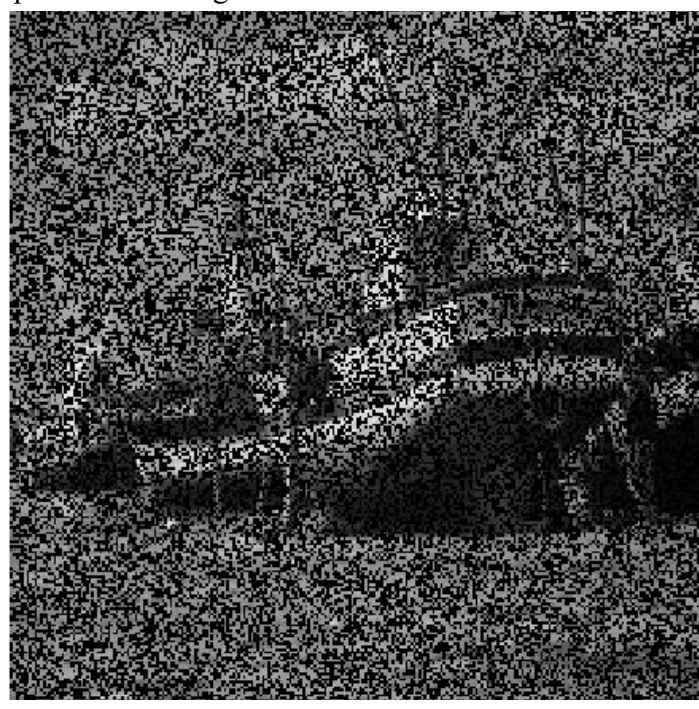

Figure 4. Comparison Picture after Noise Adding

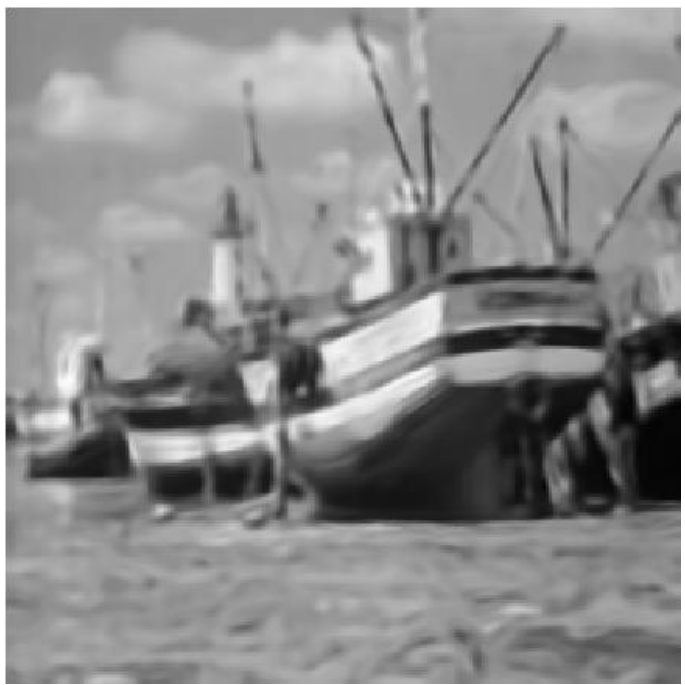

Figure 5. Image after Denoising

\section{CONCLUSION}

Among the image denoising methods, partial differential equation is a good algorithm for denoising. During 


\section{MATEC Web of Conferences}

the process of building continuous models for images, by solving the partial differential equations of the image information, changes can be made to the images by certain PDE according to the conditions of the constant values, so as to obtain better denoising images. In a manner of speaking, in the PDE processing of images, the key factor is to establish a reasonable partial differential equation in order to reach the optimal denoising effect.

\section{REFERENCES}

[1] Xiao L. 2003. Theory, Algorithm and Application of the Image Modeling Based on Variation PDE and Multi Fractal. Nanjing: Nanjing University of Science and Technology.

[2] Xia D.S. \& Fu D.S. 2004. Computer Image Processing and Its Application. Nanjing: Southeast University Press.

[3] Chen Q., Zhu L.X. \& Xia D.S.2005. Image Binaryzation in Combination with Canny Operator. Journal of Computer-Aided Design \& Computer Graphics, 27(6): 1302-2306.

[4] Chen S.S. 2008. The Image De-nosing Based on Partial Differential Equation and the Study on Quality Evaluation. Shanghai: Shanghai Jiaotong University.

[5] Wang X.P. 2007. Research of the Image De-nosing Based on Partial Differential Equation. Nanjing: Nanjing University of Science and Technology.

[6] Zhang J.P. 2012. The Image De-noising and Segmentation Methods Based on Partial Differential Equation. Dalian: Dalian University of Technology.

[7] Wang D.K., Hou Y.Q. \& Peng J.Y. 2008. The Partial Differential Equation Method of Image Processing. Science Press. 